\title{
Crescimento de Callinectes sapidus (Crustacea, Decapoda, Portunidae) no estuário da laguna dos Patos, RS, Brasil
}

\author{
Leonardo S. Ferreira ${ }^{1} \&$ Fernando D’Incao ${ }^{2}$
}

1. Programa de Pós-Graduação em Oceanografia Biológica, FURG; bolsista CNPq. (leocrab@bol.com.br)

2. Depto de Oceanografia, Fundação Universidade do Rio Grande (FURG), Av. Itália, km 78, Caixa Postal 474, 96201-900 Rio Grande, RS, Brasil.

\begin{abstract}
Growth of Callinectes sapidus (Crustacea, Decapoda, Portunidae) in the estuary of the Patos Lagoon, RS, Brazil. Using modal progression analysis for indirect age determination, growth of the blue crab Callinectes sapidus Rathbun, 1896 was estimated for two fishery areas of the Patos Lagoon. Individuals were sampled using a conventional gear of the artisanal fishery - trawl net - from February, 2005 to March, 2006 in 'Saco da Mangueira' and 'Saco do Arraial'. A total of 2,609 (1,194 males and 1,416 females) animals was caught. Growth curves were estimated using the von Bertalanffy growth model. Estimated growth curves were validated in the light of the life cycle and biological characteristics of the species. Asymptotic width (CWmax) was fixed in all analyses $(162.71 \mathrm{~mm}, \pm$ s.d.=3.10 and $157.78 \mathrm{~mm}, \pm$ s.d.=5.45 for males and females respectively), as this value is an average of the maximum lengths of crabs caught in the estuary over a period of 20 years. Growth parameters and longevity were estimated for males ('Saco da Mangueira', K=0.0039/day; $\mathrm{t}_{\mathrm{o}}=-6.07 ; 1195$ days; 'Saco do Arraial', $\mathrm{K}=0.0041 /$ day; $\mathrm{t}_{\mathrm{o}}=-5,84 ; 1102$ days) and females ('Saco da Mangueira', $K=0.0040 /$ day; $t_{0}=-6,22 ; 1153$ days; 'Saco do Arraial', $K=0.0039 /$ day; $t_{0}=-5.91$; 1181 days). Growth curves estimated in the present investigation indicate that the minimum legal size $(120 \mathrm{~mm})$ is attained around the first year of life.
\end{abstract}

KEYWORDS. Bertalanffy, Brachyura, blue crab, Patos Lagoon, trawl net.

RESUMO. Utilizando o método de deslocamento modal para a identificação das idades, estimou-se o crescimento do siri-azul Callinectes sapidus Rathbun, 1896 em duas áreas de pesca no estuário da Lagoa dos Patos. Os indivíduos foram coletados entre fevereiro de 2005 e março de 2006 no Saco da Mangueira e Saco do Arraial por meio de arrasto de rede de portas da pesca artesanal. Coletou-se um total de 2.609 animais, sendo 1.193 machos e 1.416 fêmeas. Para obtenção das curvas de crescimento utilizou-se o modelo de von Bertalanffy. As curvas foram validadas pela sua adequação ao ciclo de vida e aspectos biológicos da espécie. O tamanho máximo de largura de carapaça (LCmáx) utilizado foi mantido fixo em todas as análises (LCmáx=162,71mm; \pm d.p.=3,10 para machos e LCmáx=157,78mm; \pm d.p. $=5,45$ para fêmeas), sendo esses valores médios das maiores medidas obtidas em mais de 20 anos de coletas no estuário. Os parâmetros de crescimento e longevidade foram estimados para machos $($ Saco da Mangueira, $\mathrm{K}=0,0039 /$ dia; $\mathrm{t}=-6.07$; 1.195 dias; Saco do Arraial, $\mathrm{K}=0,0041 /$ dia; $\mathrm{t}_{\mathrm{o}}=-5,84 ; 1.102$ dias) e fêmeas (Saco da Mangueira, $\mathrm{K}=0,0040 /$ dia; $\mathrm{t}_{\mathrm{o}}=-6,22 ; 1.153$ dias; Saco do Arraial, $\mathrm{K}=0,0039 / \mathrm{dia} ; \mathrm{t}_{\mathrm{o}}=-5.91 ; 1.181$ dias). As curvas de crescimento estimadas nesse trabalho denotam que a espécie atinge o tamanho mínimo de captura praticamente no primeiro ano de vida $(120 \mathrm{~mm})$.

PALAVRAS-CHAVE. Bertalanffy, Brachyura, siri-azul, laguna dos Patos, rede-de-arrasto.

O siri-azul Callinectes sapidus Rathbun, 1896 é considerado um predador bentônico chave, controlando a diversidade, abundância e estrutura de várias comunidades bentônicas (HINEs et al., 1987). Alimentase principalmente de moluscos bivalves e gastrópodes e também de organismos em decomposição (WARNER, 1977).

A espécie tem ampla distribuição no Atlântico americano, reportada de forma disjunta desde os Estados Unidos até a Venezuela e do Rio de Janeiro (Brasil) até o norte da Argentina; também ocorre na Europa, onde foi introduzido em 1901, da Dinamarca até o sul da França, no leste do Mar Mediterrâneo e oeste do Mar Negro (WiLliams, 1974). Também é citada sua introdução no Japão (Powers, 1977).

O crescimento em tamanho das espécies do gênero foi pouco estudado no Brasil. BRAnco \& MASUnARI (1992) avaliaram o crescimento de Callinectes danae Smith, 1869 na Lagoa da Conceição em Florianópolis (Santa Catarina), enquanto que MantelatTo \& Fransozo (1999) estudaram Callinectes ornatus Ordway, 1863 na região de Ubatuba (São Paulo). O crescimento de $C$. sapidus ainda não foi abordado no Brasil, mas existem trabalhos realizados nos Estados Unidos (VAN Engel, 1958; TAgatZ, 1968; Williams, 1974; Rugolo et al., 1998).
Este trabalho tem como objetivo estudar o crescimento do siri-azul por meio do deslocamento modal dos grupos etários bem como comparar as populações de duas áreas de pesca no estuário da laguna dos Patos. Os locais foram escolhidos devido às diferenças de salinidade observadas e informações diárias de cinco pontos de coleta de salinidade, disponíveis no banco de dados do Laboratório de Crustáceos Decápodos/FURG.

\section{MATERIAL E MÉTODOS}

A laguna dos Patos é um sistema estuarino-lagunar localizado na planície costeira do Estado do Rio Grande do Sul (RS), entre $30^{\circ} 15^{\prime} \mathrm{S}, 32^{\circ} 10^{\prime} \mathrm{S}$ e $50^{\circ} 45^{\prime} \mathrm{W}, 52^{\circ} 15^{\prime} \mathrm{W}$, sendo um dos mais importantes recursos hídricos do estado (Villwock, 1978). A região estuarina da lagoa é caracterizada por apresentar enseadas rasas e numerosas ilhas (Cordazzo \& Seeliger, 1995). Possui uma área de aproximadamente $10.360 \mathrm{~km}^{2}$, drenando uma bacia de $140.000 \mathrm{~km}^{2}$, sendo considerada a maior laguna do mundo do tipo estrangulada (KJERFVE, 1986). A área sul da laguna dos Patos é uma feição do tipo estuário por apresentar características de corpo de água costeiro, fechado, com livre ligação com o mar. Porém, ressalta-se o aspecto de 
não contar com uma influência marcante de marés. Por conseguinte, o fluxo e refluxo da água do mar no interior da laguna ficam condicionados pela direção e intensidade dos ventos e pela descarga proveniente de extensa rede de drenagem do sistema laguna dos Patos - laguna Mirim (CAstello \& Möller, 1978). A área de estudo compreende o Saco da Mangueira $\left(32^{\circ} 05^{\prime} S, 5^{\circ} 08^{\prime} \mathrm{W}\right)$ e o Saco do Arraial (32 $02^{\prime} \mathrm{S}, 5^{\circ} 08^{\prime} \mathrm{W}$ ) (Fig. 1); ambos locais de amostragem possuem diferenças de salinidade.

Os exemplares de $C$. sapidus foram obtidos por meio de coletas mensais realizadas de fevereiro de 2005 a março de 2006, nos dois locais. As coletas diurnas foram efetivadas a partir de arrastos de 15 minutos com rede de portas com malha de $35 \mathrm{~mm}$ (medidas esticadas entre-nós opostos). Nas amostragens foi utilizada sempre a mesma embarcação e os arrastos foram realizados a uma velocidade de 0,25 nós. O material foi etiquetado no local e transportado para o Laboratório de Crustáceos Decápodos, Departamento de Oceanografia FURG, onde foi congelado. O processamento das amostras obedeceu ao seguinte protocolo: descongelamento em temperatura ambiente, separação por espécie e biometria; esta última etapa consistiu das seguintes fases: sexagem dos indivíduos, mensuração da largura da carapaça e da medida entre a extremidade dos maiores espinhos laterais (WILLIAMS, 1974), peso total em gramas (precisão 0,01g) e a verificação da maturidade sexual com base no abdômen.

$\mathrm{O}$ crescimento em tamanho foi estimado para machos e fêmeas por meio do acompanhamento do deslocamento modal, utilizando-se o método de máxima verossimilhança para estimar os parâmetros do modelo de VON BERTALANFFY (1938).

Para análise modal, os dados de comprimento foram agrupados em classes de tamanho de $5 \mathrm{~mm}$. Aos picos observados nas distribuições de freqüências de

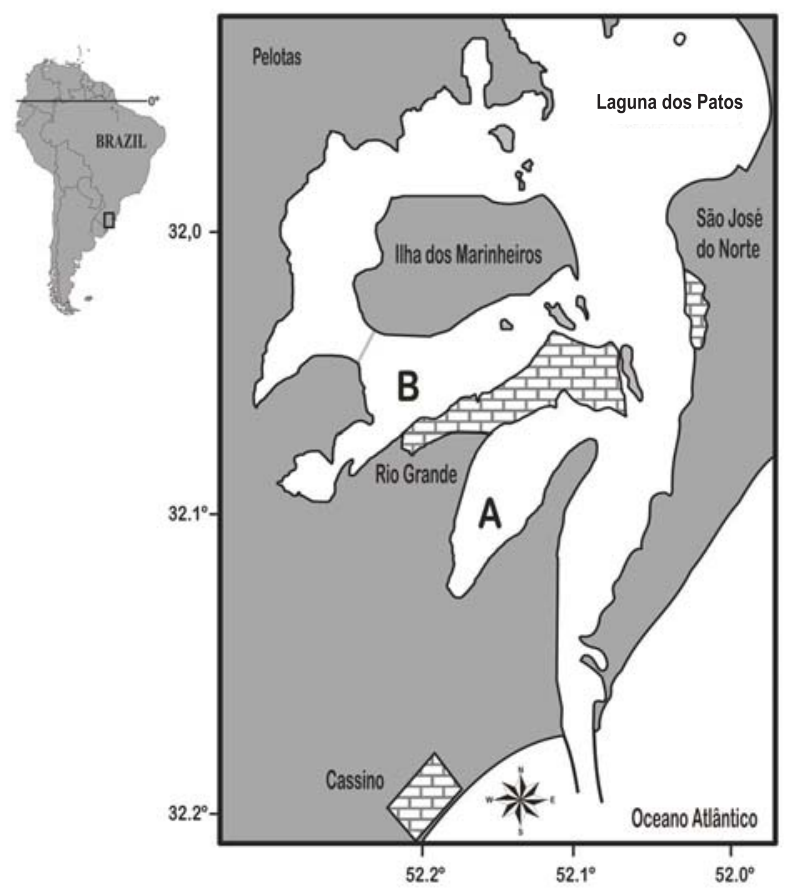

Fig. 1. Laguna dos Patos e região adjacente com as áreas de amostragem de Callinectes sapidus Rathbun, 1896. As regiões hachuradas representam áreas habitadas. A, Saco da Mangueira; B, Saco do Arraial. comprimento foram ajustadas curvas normais (curva de Gauss), de maneira que os valores da média e da moda das normais ajustadas são os mesmos.

A curva de Gauss é descrita pela equação: $\mathrm{Y}=$ $\mathrm{a}_{0}\left[\exp \left(-1 / 2\left(\mathrm{x}-\mathrm{a}_{1}\right) \mathrm{a}_{2}\right) 2\right]$, onde: $\mathrm{a}_{0}=$ amplitude da curva, $\mathrm{a}_{1}=$ média, $\mathrm{a}_{2}=$ desvio padrão.

Um resumo estatístico de cada um dos ajustes foi obtido, constando do coeficiente de determinação $\left(\mathrm{R}^{2}\right)$, os graus de liberdade (GL) e o valor de F crítico calculado. Em todos os ajustes o valor de F esteve acima do F crítico (SOKAL \& RohlF, 1969; ZAR, 1984).

As modas foram plotadas em um gráfico de dispersão contra o tempo, para o acompanhamento do ritmo de crescimento das coortes. Os parâmetros de crescimento foram calculados para as diferentes coortes de maneira interativa, através da ferramenta "Solver" (Office XP) a qual procura, a partir de valores "sementes", minimizar as somas dos resíduos entre os comprimentos observados em campo e os calculados pelo modelo de VON BERTALANFFy (1938), variando os parâmetros ( $\mathrm{k} \mathrm{e} \mathrm{t}_{0}$ ) da equação.

A largura de carapaça máxima (LCmáx) utilizada foi uma média dos maiores valores de tamanho já coletados no estuário da Laguna dos Patos (banco de dados do Laboratório de Crustáceos Decápodos/FURG) para uma série histórica de mais de 20 anos. Os valores fixados na análise foram de $162,71 \mathrm{~mm} \pm$ d.p. $=3,10$ para machos e $157,78 \mathrm{~mm} \pm$ d.p. $=5,45$ para fêmeas. Este parâmetro foi mantido fixo na análise, permitindo-se a variação de $\mathrm{t}_{0} \mathrm{e} \mathrm{k}$.

Foram selecionadas as coortes que apresentaram ritmo de crescimento coerente quanto à longevidade, coeficiente de crescimento e com coeficiente de determinação $\left(\mathrm{R}^{2}\right)$ alto ( $\mathrm{t}[0,80)$. As curvas foram consideradas a partir do tamanho do primeiro estágio juvenil (crab I, idade 0) que, segundo BARUTOT et al. (2001), é igual a 2,61 mm excluídas as fases larvais.

Depois de selecionadas as modas, foram realizadas as correções das idades em dias, baseadas nos intervalos de tempo entre as coletas e no valor de $\mathrm{t}_{0}$ encontrado. Os comprimentos modais e as idades corrigidas foram utilizados para o cálculo de uma curva de crescimento média, através da equação de voN BERTALANFFy (1938):

$\mathrm{LCt}=\mathrm{LC}$ क $\left[1-\mathrm{e}-\mathrm{k}\left(\mathrm{t}-\mathrm{t}_{0}\right)\right]$, onde $\mathrm{LCt}=$ comprimento total no tempo $\mathrm{t}, \mathrm{k}=$ constante de crescimento, $\mathrm{t}_{0}=$ parâmetro de ajuste (representando a idade do indivíduo quando seu tamanho é igual a zero).

As curvas médias obtidas para machos e fêmeas de ambos os locais foram analisadas estatisticamente para comparação do crescimento entre os sexos. Para estas análises, utilizou-se o teste $\mathrm{F}$ (teste de hipótese) com significância de 5\% (CERRATO, 1990).

A longevidade de 2,5 a 3 anos obtida por WiLliams (1974) foi considerada como valor de comparação para a aceitação das coortes estimadas. Foram consideradas aquelas estimativas cuja longevidade se situou em torno destes valores.

Nesse trabalho obteve-se a longevidade por meio da equação inversa de vON BERTALANFFY (1938), adaptada por D'INCAO \& FONSECA (1999):

$\mathrm{T}_{\text {máx }}=\left(0-1(1 / \mathrm{k}) \operatorname{Ln}(1-\mathrm{LCt} / \mathrm{LC} \infty)\right.$, onde $\mathrm{T}_{\text {máx }}=$ longevidade. 


\section{RESULTADOS}

Foi coletado um total de 2.609 indivíduos, sendo 1.193 machos $(45,7 \%)$ e 1.416 fêmeas $(54,3 \%)$. No Saco da Mangueira foram capturados 1.258 indivíduos, 574 machos com largura da carapaça entre 3,12 e 15,51 cm e 684 fêmeas (2,85 e 14,26cm). No Saco do Arraial o número total de exemplares foi 1351 - 619 machos com 2,41 a 15,55cm, e 732 fêmeas entre 2,69 a 14,13cm de largura.

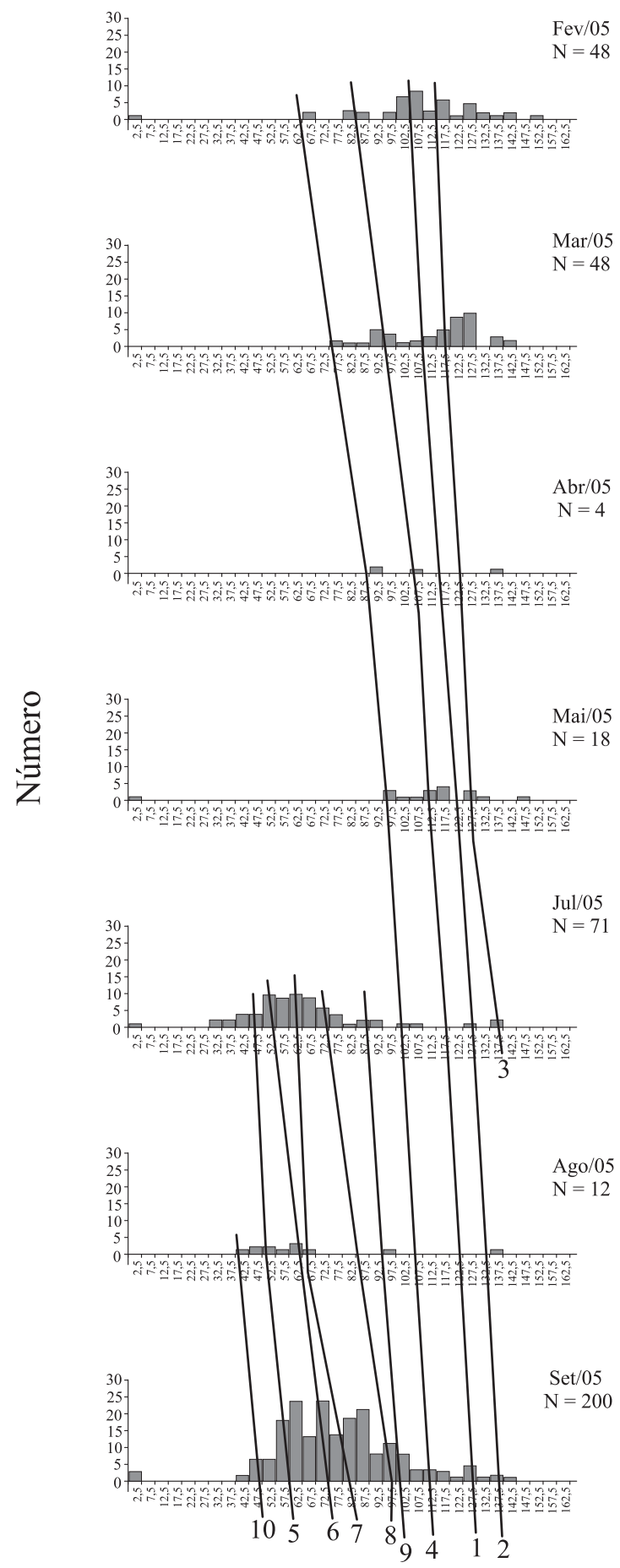

As distribuições de frequiência de comprimento são polimodais, refletindo as diferentes coortes que se incorporam à população ao longo do tempo (Figs. 2-5). Foram selecionadas para a análise das curvas de crescimento dez coortes para machos (Fig. 2) e quinze para fêmeas (Fig. 3) no Saco da Mangueira e onze coortes para machos (Fig. 4) e doze para fêmeas (Fig. 5) no Saco do Arraial. As tabelas I e II mostram os parâmetros de crescimento estimados para ambos os sexos nos dois locais de coleta.

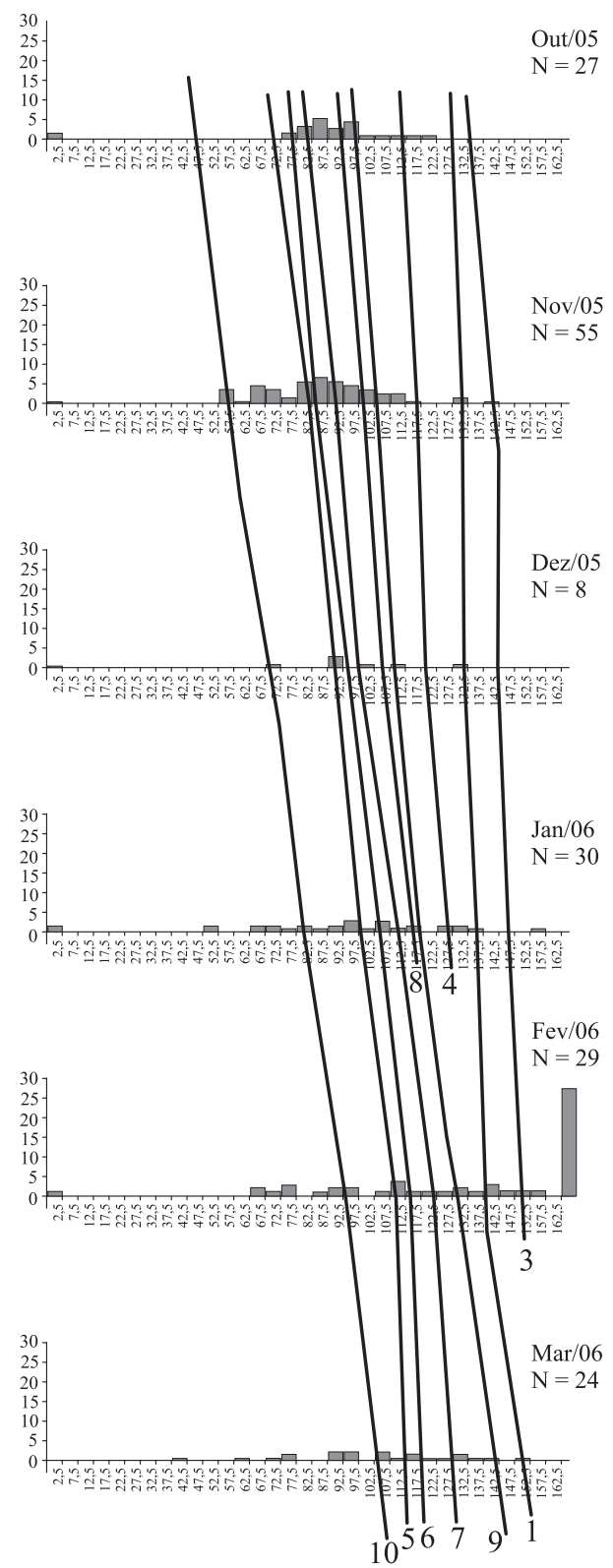

\section{Classes de tamanho}

Fig. 2. Análise da progressão modal de machos de Callinectes sapidus Rathbun, 1896 coletados no Saco da Mangueira, estuário da laguna dos Patos, de fevereiro de 2005 a março de 2006. As linhas representam as coortes seguidas ao longo do tempo para descrever o crescimento individual. 
As curvas gerais para cada sexo foram estimadas para ambos locais de coleta (Tab. III; Figs. 6, 7). A longevidade estimada foi de 3,27 anos (1.195 dias) e 3,16 anos (1.153 dias) anos para machos e para fêmeas respectivamente no Saco da Mangueira, enquanto que para o Saco do Arraial foi de 3,02 anos (1.102 dias) e 3,24 anos (1.181 dias).

Os valores de $\mathrm{R}^{2}$ foram elevados, mantendo-se em
0,99 para todas as estimativas. O teste $\mathrm{F}$ para a comparação entre as curvas de crescimento entre machos mostrou diferença significativa $\left(\mathrm{F}_{\text {calculado }}=21,46 ; \mathrm{F}_{\text {tabelado }}=\right.$ $3,90)$, sendo os machos do Saco da Mangueira maiores que os do Saco do Arraial. Quando comparadas às curvas de crescimento das fêmeas, não mostraram diferenças estatísticas significativas $\left(\mathrm{F}_{\text {calculado }}=0,04 ; \mathrm{F}_{\text {tabelado }}=3,89\right)$.

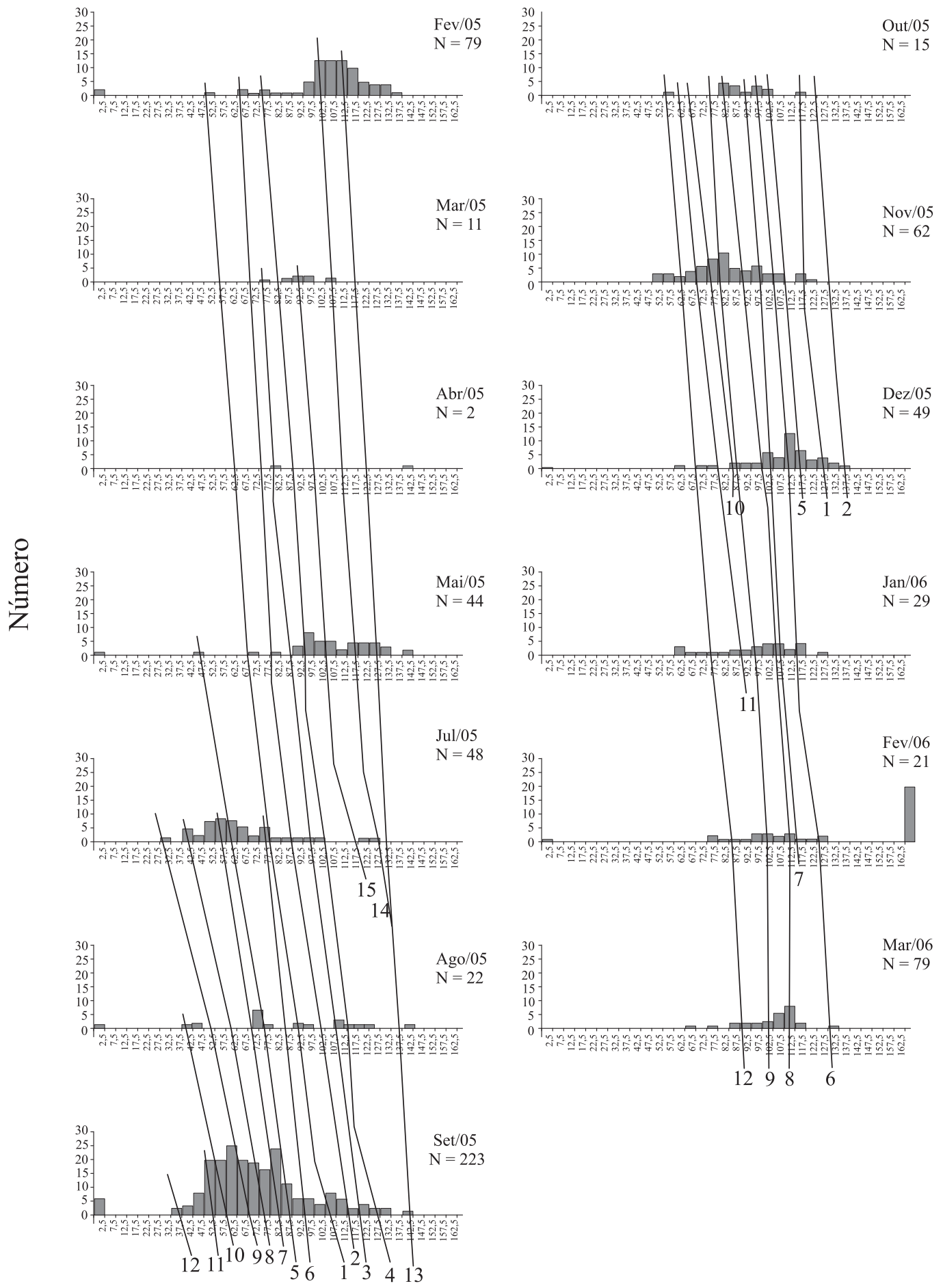

\section{Classes de tamanho}

Fig. 3. Análise da progressão modal de fêmeas de Callinectes sapidus Rathbun, 1896 coletados no Saco da Mangueira, estuário da laguna dos Patos, de fevereiro de 2005 a março de 2006. As linhas representam as coortes seguidas ao longo do tempo para descrever o crescimento individual. 
Tabela I. Parâmetros de crescimento e ajuste das coortes de machos e fêmeas de Callinectes sapidus Rathbun, 1896 coletados no Saco da Mangueira durante o período de fevereiro de 2005 a março de 2006 no estuário da laguna dos Patos, RS (LC $\infty$, tamanho máximo encontrado $(\mathrm{mm}) ; \mathrm{K}$, constante de crescimento; $\mathrm{t}_{0}$, tamanho na idade zero; $\mathrm{t}_{\max }$, longevidade máxima (em anos); $\mathrm{R}^{2}$, coeficiente de correlação).

\begin{tabular}{|c|c|c|c|c|c|c|c|c|c|c|}
\hline \multicolumn{6}{|c|}{ Machos } & \multicolumn{5}{|c|}{ Fêmeas } \\
\hline & $\mathrm{LC} \infty$ & $\mathrm{K}$ & $\mathrm{t}_{0}$ & $\mathrm{t}_{\text {máx }}$ & $\mathrm{R}^{2}$ & $\mathrm{LC} \infty$ & $\mathrm{K}$ & $\mathrm{t}_{0}$ & $\mathrm{t}_{\text {máx }}$ & $\mathrm{R}^{2}$ \\
\hline Coorte 1 & 162,71 & 0,004 & $-192,1$ & 3,14 & 0,97 & 157,78 & 0,003 & $-97,6$ & 3,49 & 0,99 \\
\hline Coorte 2 & 162,71 & 0,004 & $-245,2$ & 3,01 & 1,00 & 157,78 & 0,003 & $-137,7$ & 3,47 & 0,99 \\
\hline Coorte 3 & 162,71 & 0,004 & $-320,0$ & 3,17 & 1,00 & 157,78 & 0,003 & $-176,8$ & 3,53 & 0,99 \\
\hline Coorte 4 & 162,71 & 0,003 & $-183,5$ & 3,94 & 0,97 & 157,78 & 0,004 & $-155,2$ & 3,00 & 0,99 \\
\hline Coorte 5 & 162,71 & 0,004 & $-72,9$ & 3,32 & 1,00 & 157,78 & 0,004 & $-153,6$ & 2,88 & 1,00 \\
\hline Coorte 6 & 162,71 & 0,004 & $-86,51$ & 3,06 & 1,00 & 157,78 & 0,004 & $-73,2$ & 2,82 & 0,99 \\
\hline Coorte 7 & 162,71 & 0,004 & $-108,5$ & 3,15 & 1,00 & 157,78 & 0,004 & $-113,3$ & 3,12 & 0,96 \\
\hline Coorte 8 & 162,71 & 0,004 & $-156,6$ & 3,39 & 1,00 & 157,78 & 0,004 & $-83,2$ & 3,07 & 0,97 \\
\hline Coorte 9 & 162,71 & 0,004 & $-178,2$ & 2,99 & 0,99 & 157,78 & 0,004 & $-72,9$ & 3,42 & 0,94 \\
\hline Coorte 10 & 162,71 & 0,003 & $-63,7$ & 3,65 & 0,99 & 157,78 & 0,004 & $-74,4$ & 2,95 & 0,99 \\
\hline Coorte 11 & & & & & & 157,78 & 0,004 & $-114,3$ & 3,37 & 0,99 \\
\hline Coorte 12 & & & & & & 157,78 & 0,003 & $-96,2$ & 3,79 & 0,97 \\
\hline Coorte 13 & & & & & & 157,78 & 0,004 & $-314,5$ & 2,97 & 1,00 \\
\hline Coorte 14 & & & & & & 157,78 & 0,004 & $-239,8$ & 2,83 & 0,99 \\
\hline Coorte 15 & & & & & & 157,78 & 0,004 & $-225,7$ & 3,13 & 0,99 \\
\hline
\end{tabular}

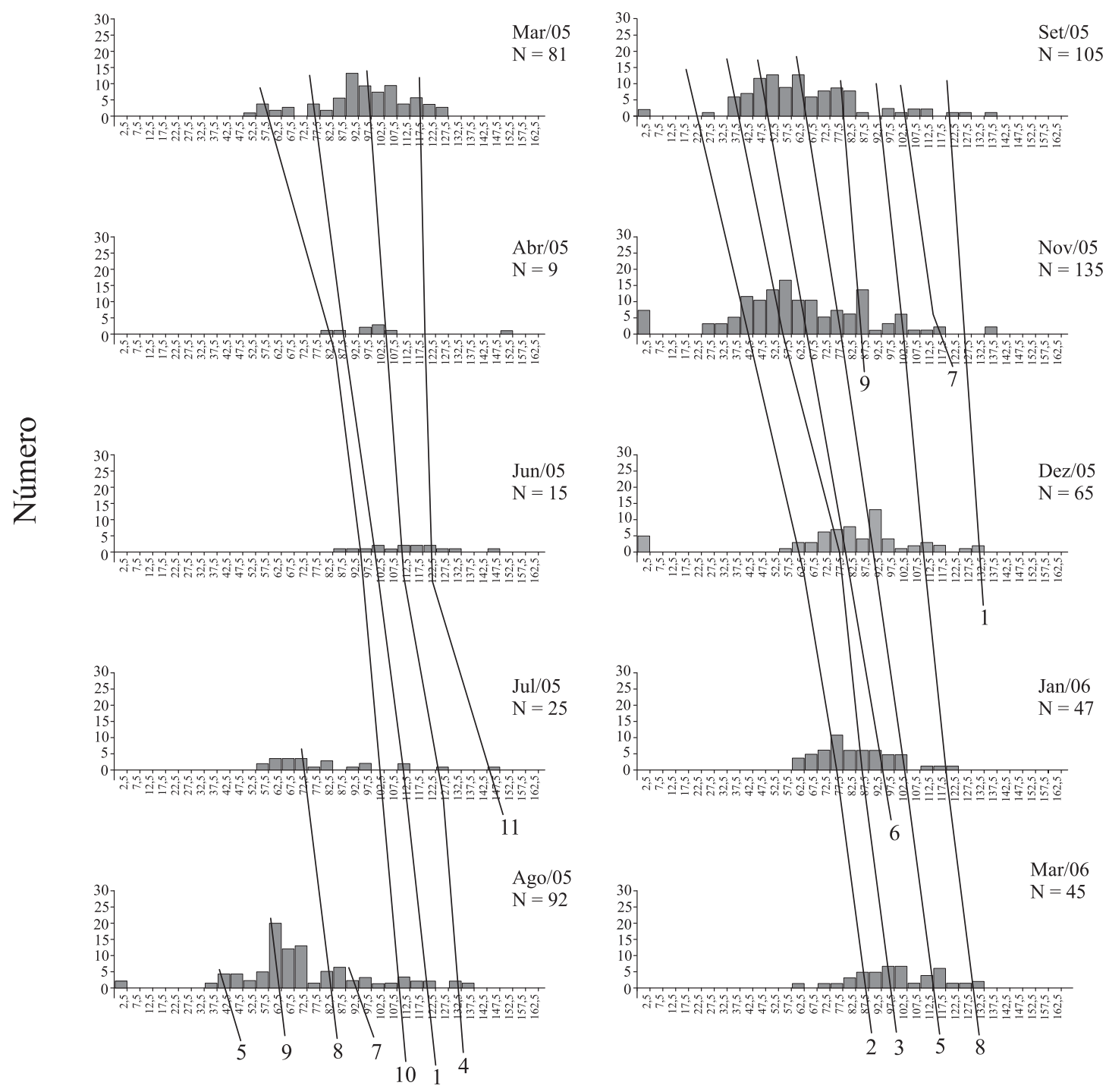

Classes de tamanho

Fig. 4. Análise da progressão modal de machos de Callinectes sapidus Rathbun, 1896 coletados no Saco do Arraial, estuário da laguna dos Patos, de fevereiro de 2005 a março de 2006. As linhas representam as coortes seguidas ao longo do tempo para descrever o crescimento individual. 
Tabela II. Parâmetros de crescimento e ajuste das coortes de machos e fêmeas de Callinectes sapidus Rathbun, 1896 coletados no Saco do Arraial durante o período de março de 2005 a março de 2006 no estuário da laguna dos Patos, RS (LC $\infty$, tamanho máximo encontrado $(\mathrm{mm}) ; \mathrm{K}$, constante de crescimento; $\mathrm{t}_{0}$, tamanho na idade zero; $\mathrm{t}_{\max }$, longevidade máxima (em anos); $\mathrm{R}^{2}$, coeficiente de correlação).

\begin{tabular}{|c|c|c|c|c|c|c|c|c|c|c|}
\hline & \multicolumn{5}{|c|}{ Machos } & \multicolumn{5}{|c|}{ Fêmeas } \\
\hline & $\mathrm{LC} \infty$ & $\mathrm{K}$ & $\mathrm{t}_{0}$ & $\mathrm{t}_{\text {máx }}$ & $\mathrm{R}^{2}$ & $\mathrm{LC}_{\infty}$ & $\mathrm{K}$ & $\mathrm{t}_{0}$ & $\mathrm{t}_{\text {máx }}$ & $\mathrm{R}^{2}$ \\
\hline Coorte 1 & 162,71 & 0,004 & $-164,6$ & 3,28 & 0,97 & 157,78 & 0,004 & $-152,2$ & 3,24 & 0,99 \\
\hline Coorte 2 & 162,71 & 0,004 & $-48,8$ & 3,37 & 0,98 & 157,78 & 0,003 & $-198,3$ & 3,65 & 1,00 \\
\hline Coorte 3 & 162,71 & 0,004 & $-70,5$ & 3,16 & 0,99 & 157,78 & 0,004 & $-192,4$ & 2,77 & 1,00 \\
\hline Coorte 4 & 162,71 & 0,004 & $-223,4$ & 3,06 & 0,99 & 157,78 & 0,004 & $-309,2$ & 2,87 & 0,99 \\
\hline Coorte 5 & 162,71 & 0,004 & $-73,6$ & 2,85 & 0,98 & 157,78 & 0,004 & $-178,5$ & 3,03 & 0,99 \\
\hline Coorte 6 & 162,71 & 0,004 & $-82,3$ & 2,82 & 1,00 & 157,78 & 0,004 & $-129,5$ & 3,03 & 0,98 \\
\hline Coorte 7 & 162,71 & 0,005 & $-194,4$ & 2,67 & 0,99 & 157,78 & 0,004 & $-147,2$ & 3,34 & 0,97 \\
\hline Coorte 8 & 162,71 & 0,004 & $-147,8$ & 3,10 & 0,99 & 157,78 & 0,003 & $-105,9$ & 3,56 & 0,94 \\
\hline Coorte 9 & 162,71 & 0,004 & $-136,6$ & 3,43 & 0,99 & 157,78 & 0,004 & $-109,0$ & 3,04 & 1,00 \\
\hline Coorte 10 & 162,71 & 0,004 & $-105,1$ & 2,90 & 0,98 & 157,78 & 0,004 & $-63,2$ & 3,06 & 0,98 \\
\hline Coorte 11 & 162,71 & 0,005 & $-278,1$ & 2,76 & 0,83 & 157,78 & 0,003 & $-76,2$ & 3,85 & 0,91 \\
\hline Coorte 12 & & & & & & 157,78 & 0,003 & $-152,8$ & 3,79 & 1,00 \\
\hline
\end{tabular}
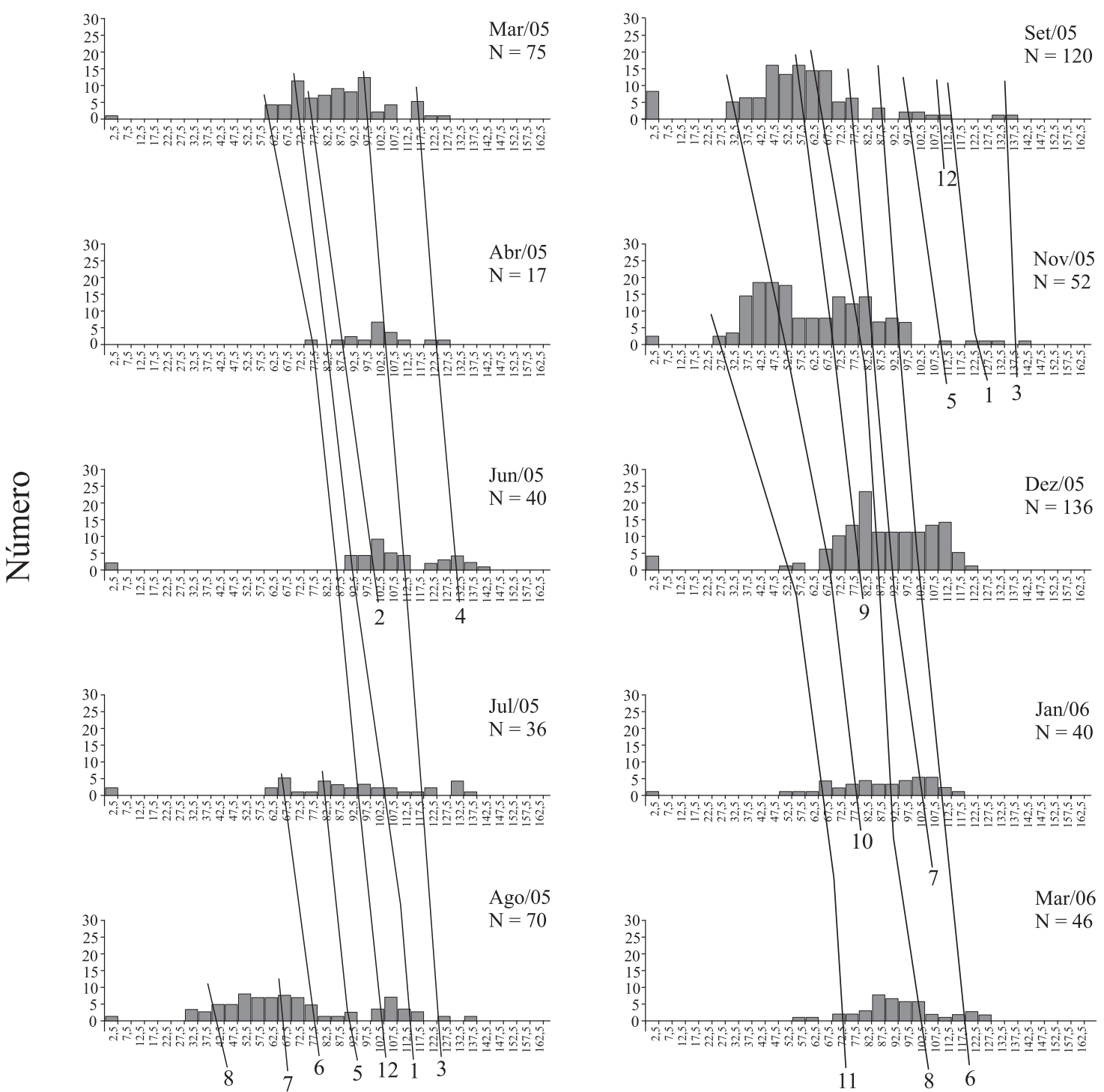

Classes de tamanho

Fig. 5. Análise da progressão modal de fêmeas de Callinectes sapidus Rathbun, 1896 coletados no Saco do Arraial, estuário da laguna dos Patos, de fevereiro de 2005 a março de 2006. As linhas representam as coortes seguidas ao longo do tempo para descrever o crescimento individual. 
Tabela III. Sumário das estimativas da curva de crescimento em largura de carapaça segundo von Bertalanffy, seus parâmetros de ajuste e longevidade estimada para o Saco da Mangueira (machos e fêmeas) e para o Saco do Arraial (machos e fêmeas) de Callinectes sapidus Rathbun, 1896 capturados no estuário da laguna dos Patos. (LC $\propto$ tamanho máximo encontrado (mm); $\mathrm{K}$, constante de crescimento; $\mathrm{t}_{0}$, tamanho na idade zero).

\begin{tabular}{|c|c|c|c|c|c|}
\hline Saco da Mangueira & $\mathrm{LC}_{\infty}(\mathrm{mm})$ & $\mathrm{K}(/ \mathrm{dia})$ & $\mathrm{t}_{0}(/$ dias $)$ & Equação & $\begin{array}{c}\text { Longevidade máx } \\
\text { (dias; anos) }\end{array}$ \\
\hline Machos & 162,71 & 0,0039 & $-6,07$ & $\mathrm{LC}=162,71 *(1-\mathrm{e}(0,0039 *(\mathrm{t}-6,07)))$ & $1.194 ; 3,27$ \\
\hline Fêmeas & 157,78 & 0,0040 & $-6,22$ & $\mathrm{LC}=157,78 *(1-\mathrm{e}(0,0040 *(\mathrm{t}-6,22)))$ & $1.185 ; 3,16$ \\
\hline Saco do Arraial & $\mathrm{LC}_{\infty}(\mathrm{mm})$ & K(/dia) & $\mathrm{t}_{0}(/ \mathrm{dias})$ & Equação & $\begin{array}{l}\text { Longevidade máx } \\
\text { (dias; anos) }\end{array}$ \\
\hline Machos & 162,71 & 0,0041 & $-5,84$ & $\mathrm{LC}=162,71 *(1-\mathrm{e}(0,0041 *(\mathrm{t}-5,84)))$ & $1.110 ; 3,02$ \\
\hline Fêmeas & 157,78 & 0,0039 & $-5,91$ & $\mathrm{LC}=157,78 *(1-\mathrm{e}(0,0039 *(\mathrm{t}-5,91)))$ & $1.182 ; 3,24$ \\
\hline
\end{tabular}

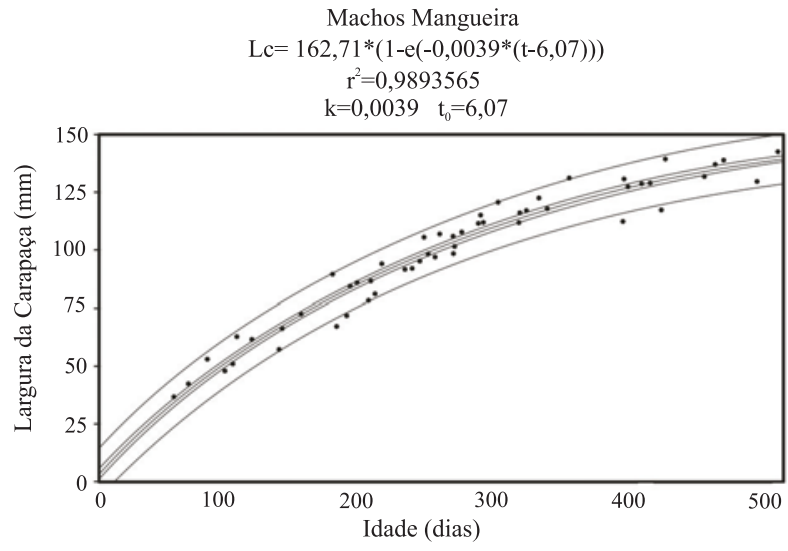

Fêmeas Mangueira

$\mathrm{Lc}=157,78 *(1-\mathrm{e}(-0,0040 *(\mathrm{t}-6,22)))$

$\mathrm{r}^{2}=0,98827159$

$\mathrm{k}=0,0040 \quad \mathrm{t}_{0}=6,22$

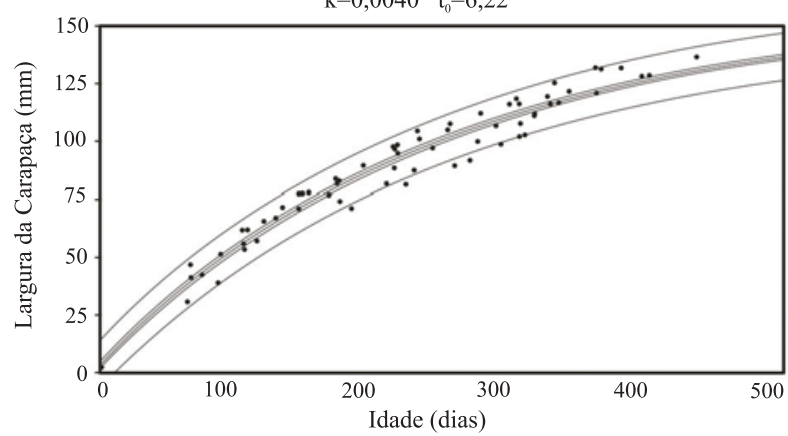

Fig. 6. Curvas de crescimento e parâmetros da equação de von Bertalanffy estimados separadamente para machos e fêmeas de Callinectes sapidus Rathbun, 1896 coletados no Saco da Mangueira, estuário da laguna dos Patos. A linha central é a média, as linhas externas são os intervalos de predição (95\%) e as internas são os intervalos de confiança estimados (95\%).

\section{DISCUSSÃO}

O modelo de von Bertalanffy mostrou-se adequado em descrever o crescimento de $C$. sapidus para os dados coletados. Este modelo já foi utilizado com sucesso no estudo de crescimento de juvenis e adultos de camarãorosa (D'INCAO, 1984). BRANCO \& MASUNARI (1992) utilizaram a rotina Elefan (FISAT) para encontrar os parâmetros de crescimento de $C$. danae. Este método realiza, por meio de uma rotina matemática, a escolha das coortes através do melhor coeficiente de ajuste. No presente estudo, o método utilizado procurou validar e valorizar parâmetros de ajuste da curva de crescimento condizentes com o ciclo de vida e biologia da espécie.
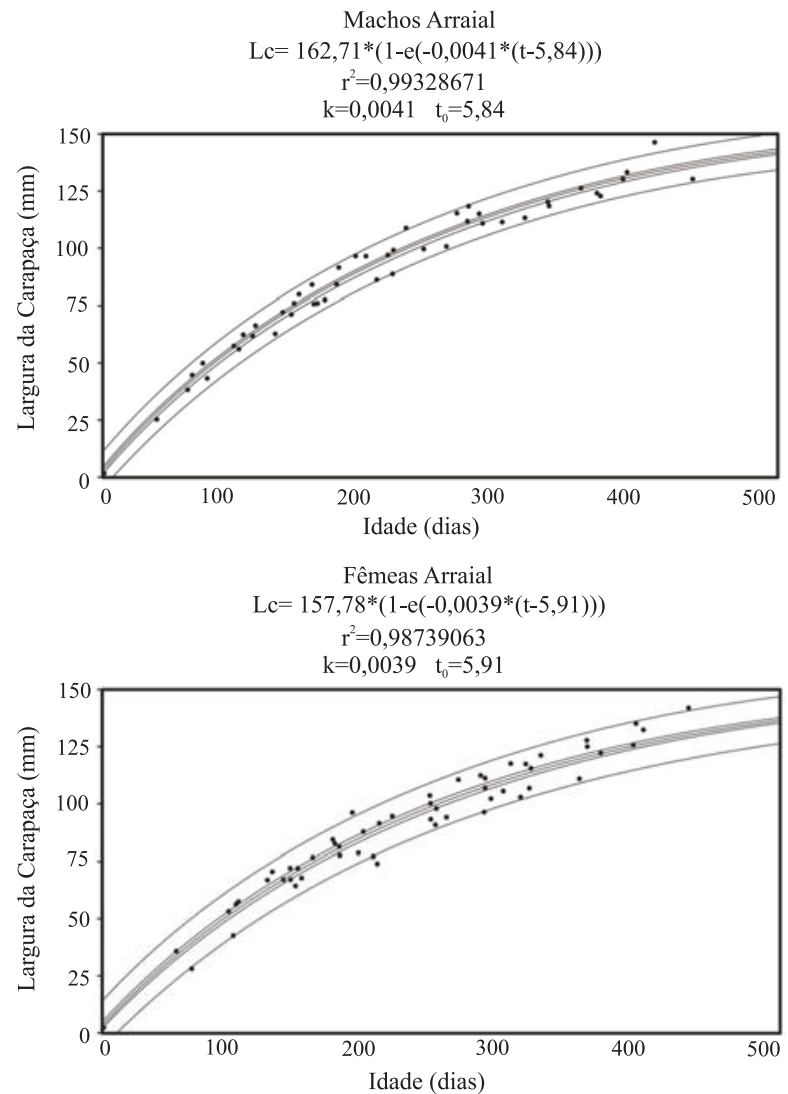

Fig. 7 Curvas de crescimento e parâmetros da equação de von Bertalanffy estimados separadamente para machos e fêmeas de Callinectes sapidus Rathbun, 1896 coletados no Saco do Arraial, estuário da laguna dos Patos. A linha central é a média, as linhas externas são os intervalos de predição $(95 \%)$ e as internas são os intervalos de confiança estimados $(95 \%)$.

O siri-azul apresenta múltiplas coortes por ano, com crescimento rápido no início do ciclo de vida e que vai diminuindo ao longo do tempo. MANTELATTO \& FRANSOZO (1999) relataram comportamento semelhante para C. ornatus.

Mesmo sendo de locais de coleta distintos, não se considerou os indivíduos como pertencentes a populações diferentes por não existirem barreiras geográficas entre os dois locais e por não terem sido realizados estudos sobre a genética desses indivíduos. No entanto, foram observadas pequenas diferenças nos parâmetros estimados, provavelmente, devido às peculiaridades do habitat em cada área considerada.

A longevidade de 2,5 a 3 anos citada por WILLIAMS (1974) é um pouco menor que a estimada para os dois locais 
de coleta. Esta diferença pode estar relacionada à diferença das populações, bem como pelo método utilizado para a escolha das curvas de crescimento, já que WiLLiams (1974) valeu-se da observação do ciclo de vida da espécie, enquanto que neste trabalho a estimativa foi feita pela equação de crescimento levando em consideração $99 \%$ dos tamanhos máximos (LC $\infty$ ) (D'INCAO \& FONSECA, 1999). VAN ENGEL (1958), em trabalho realizado em Chesapeake Bay, EUA, sugere que $C$. sapidus vive entre 3 e 3,5 anos, mas a média de vida seria de 1 ano após atingida a idade adulta. Em St. Johns River (EUA), TAGATz (1968) observou que relativamente poucos indivíduos de $C$. sapidus vivem mais que um ano após a maturidade e a idade máxima possível foi estimada em 4 anos. Rugolo et al. (1998) estimou em 8 anos a longevidade em "Chesapeake Bay", fugindo consideravelmente dos valores estimados pelos demais autores. Os valores obtidos para a laguna dos Patos, em ambas áreas, são coerentes com a maioria das estimativas registradas na literatura, mesmo que estas tenham sido para populações da América do Norte.

As curvas de crescimento estimadas nesse trabalho permitem afirmar que a espécie atinge o tamanho mínimo de captura, praticamente, no primeiro ano de vida (120mm). Este fato é importante para as populações por aumentar a probabilidade dos indivíduos reproduzirem antes de serem capturados.

Apesar da largura máxima (LC $\infty)$ ter se mantida fixa em todas as análises, o parâmetro $\mathrm{K}$ ainda pode fornecer informações importantes para o crescimento. $\mathrm{O}$ valor de $\mathrm{K} /$ anual encontrado para os machos do Saco da Mangueira foi de 1,40, enquanto que para o Saco do Arraial foi de 1,48; para as fêmeas, os valores de K/anual obtidos foram de 1,44 para o Saco da Mangueira e de 1,42 para o Saco do Arraial.

O teste $\mathrm{F}$ (CERRATO, 1990), aplicado para a comparação das curvas de crescimento entre os machos dos dois locais, apresentou diferença significativa, sendo os do Saco da Mangueira maiores que os do Saco do Arraial. Isso pode ser explicado pelo fato dos indivíduos do primeiro viverem em uma área mais protegida, por ser essa uma enseada semifechada (AlmEIDA et al., 1993). Para as fêmeas, esse teste não apresentou diferença significativa, podendo ser resultado da migração para o oceano no momento da desova. Este fator pode justificar a ausência de indivíduos maiores na área de amostragem e prejudicar a comparação.

\section{CONCLUSÕES}

O modelo de von BerTALANFFy (1938) mostrou-se adequado em descrever o crescimento do siri-azul no estuário da laguna dos Patos, a partir da fase juvenil. Callinectes sapidus apresenta múltiplas coortes por ano, com crescimento rápido no início do ciclo de vida e que vai diminuindo ao longo do tempo.

As curvas de crescimento estimadas nesse trabalho permitem verificar que a espécie atinge o tamanho mínimo de captura $(120 \mathrm{~mm})$ praticamente no primeiro ano de vida, sendo esse fator importante para as populações, por aumentar a probabilidade dos indivíduos reproduzirem antes de serem capturados.

Agradecimentos. À Secretaria de Ciência e Tecnologia do Estado Rio Grande do Sul, pelo suporte financeiro através do Programa de Pólos Tecnológicos. Ao Prof. Dr. Fernando L. M.
Mantelatto (USP) pelas importantes sugestões. Ao Dr. Duane B. Fonseca pelo auxílio no decorrer do trabalho.

\section{REFERÊNCIAS BIBLIOGRÁFICAS}

Almeida, M. T.; Baumgarten, M. G. Z. \& Rodrigues, R. M. S. 1993. Identificação das possíveis fontes de contaminação das águas que margeiam a cidade do Rio Grande-RS. Rio Grande, FURG, Documento Técnico Oceanografia, 6. $30 \mathrm{p}$.

Barutot, R. A.; Vieira, R. R. R. \& Rieger, P. J. 2001 Desenvolvimento juvenil de Callinectes sapidus Rathbun, 1896 (Crustacea: Decapoda: Portunidae), em laboratório, a partir de megalopas coletadas no plâncton. Comunicações do Museu de Ciências e Tecnologia PUCRS, Série Zoologia, 14(1):23-42.

Branco, J. O. \& Masunari, S. 1992. Crescimento de Callinectes danae Smith (Decapoda, Portunidae) da Lagoa da Conceição, Florianópolis, Santa Catarina, Brasil. Revista Brasileira de Zoologia 9(1/2):53-66.

Castelo, J. P. \& Möller, O. O. 1978. On the relationship between rainfall and shrimp production in the estuary of the Patos Lagoon (Rio Grande do Sul, Brasil). Atlântica 3:67-74.

Cerrato, R. M. 1990. Interpretable statistical tests for growth comparisons using parameters in the von Bertalanffy equation. Canadian Journal of Fisheries and Aquatic Sciences 47:1416-1426.

Cordazzo, C. V. \& Seeliger, U. 1995. Guia ilustrado da vegetação costeira no extremo sul do Brasil. Rio Grande, FURG. $275 \mathrm{p}$.

D'Incao, F. 1984. Estudo sobre o crescimento de Penaeus (Farfantepenaeus) paulensis Perez Farfante, 1967 da Lagoa dos Patos, RS, Brasil (Decapoda, Penaeidae). Atlântica 13 (1): $159-169$.

D'Incao, F. \& Fonseca, D. B. 1999. Performance of the von Bertalanffy growth curve in penaeid shrimps: A critical approach. In: Klein, J. C. von \& Schram, F. R. eds. The biodiversity crisis and Crustacea: proceedings of the Fourth International Crustacean Congress. Rotterdam, A. A. Balkema. p.733-737.

Hines, A. H.; Lipcius, R. N. \& Haddon, A. M. 1987. Population dynamics and habitat partitioning by size, sex and molt stage of blue crab Callinectes sapidus, in a subestuary of Central Chesapeake Bay. Marine Ecology Progress Series 36:55-64.

KJERFVE, B. 1986. Comparative oceanography of coastal lagoons. In: Wolfe, D. A. ed. Estuarine variability. New York Academic. p.63-81.

Mantelatto, F. L. \& Fransozo, A. 1999. Reproductive biology and molting cycle of the crab Callinectes ornatus (Decapoda, Portunidae) from the Ubatuba Region, São Paulo, Brazil. Crustaceana 72(1):64-75.

Powers, L. W. 1977. A catalogue and bibliography to the crabs (Brachyura) of the Gulf of México. Contributions in Marine Science 20:1-190.

Rugolo, L. J.; Knotts, K. S.; Lange, A. M. \& Crecco, V. A. 1998. Stock assessment of Chesapeake Bay blue crab (Callinectes sapidus Rathbun). Journal of Shellfish Research 17(2):493-517.

Sokal, R. R. \& Rohlf, F. J. 1969. Biometry: The principles and practice of statistics in biological research. San Francisco, W. H. Freeman and Company. 776p.

Tagatz, M. E. 1968. Biology of the blue crab, Callinectes sapidus Rathbun, in the St. Johns River, Florida. Fishery Bulletin 67(1):17-33.

VAn Engel, W. A. 1958. The blue crab and its fishery in Chesapeake Bay. Part 1. Reproduction, early development, growth and migration. Commercial Fisheries Review 20(6):6-17.

VILLWOCK, J. A. 1978. Aspectos da sedimentação da região nordeste da Lagoa dos Patos: Lagoa do Casamento e Saco do Cocuruto. Pesquisas 11:193-223.

von Bertalanffy, L. 1938. A quantitative theory of organic growth (inquiries on growth laws). Human Biology 10(2): $181-213$.

WARner, G. F. 1977. The biology of crabs. London, Elek Science. 202p.

Williams, A. B. 1974. The swimming crabs of the genus Callinectes (Decapoda: Portunidae). Fishery Bulletin 72(3):685-798.

Zar, J. H. 1984. Biostatiscal Analysis. London, Prentice-Hall International Editions. 718p. 